\title{
Prioridades de conservación aplicando información filogenética y endemicidad: un ejemplo basado en Carabidae (Coleoptera) de América del Sur austral
}

\author{
Conservation priorities using phylogenetic information and endemicity: an example \\ based on Carabid beetles from Southern South America
}

\author{
SERGIO ROIG-JUÑENT \& GUILLERMO DEBANDI
}

Laboratorio de Entomología, Instituto Argentino de Investigaciones en Zonas Áridas, Avenida Dr. Adrián Ruiz Leal s/n, Casilla de Correo 507, 5500 Mendoza, Argentina Autor para correspondencia: e- mail: saroig@lab.cricyt.edu.ar

\begin{abstract}
RESUMEN
Sobre la base de la información distribucional de las especies de Carabidae (Coleoptera) de América del Sur austral, se reconocieron 17 áreas de endemismo. Para cada una de ellas se estimó la diversidad gamma, el porcentaje de endemismo, el valor filogenético y la complementariedad, exceptuando Juan Fernández del cual no se pudo obtener ningún valor filogenético. El cálculo del valor filogenético se realizó a través del uso de cuatro índices, el valor filogenético sin ninguna modificación (W), considerando la endemicidad (We), y sus respectivas estandarizaciones (Ws y Wes). Los resultados muestran que la estandarización del valor filogenético (Ws) produce un sesgo cuando los cladogramas difieren en tamaño y que el complemento entre áreas es una herramienta secundaria de gran utilidad. Sin embargo, los resultados muestran que para determinar la importancia de las áreas, el complemento debe ser usado en conjunto con la diversidad específica y la endemicidad. Las comparaciones de los resultados obtenidos usando W, We y análisis de complementariedad estrictos y modificados muestran que el valor filogenético que admite endemicidad (We) puede ser aplicado como único valor para determinar la importancia de cada área. Aplicando We, las seis primeras áreas seleccionadas acumulan el $72 \%$ del valor filogenético y el $74 \%$ de la diversidad gamma de los carábidos de América del Sur austral, mientras que ninguno de los otros parámetros usados acumula el $70 \%$ de las especies antes de sumar la séptima área. Las seis áreas seleccionadas son la Selva valdiviana, las Sierras pampeanas, Coquimbo, Patagonia occidental, Chile central y La Araucanía. Es de hacer notar que las Sierras pampeanas y la Patagonia occidental son ambientes de pastizales, Coquimbo y Chile central son ambientes áridos y semiáridos, y solo la Selva valdiviana y Araucanía son típicos bosques australes.
\end{abstract}

Palabras clave: índices de valor filogenéticos, endemicidad, complementariedad, Carabidae, América del Sur.

\begin{abstract}
Based on information of the Southern South American carabid species, 17 areas of endemism were defined. Gamma diversity, percentage of endemism, phylogenetic value, and complementarity, were estimated for each area of endemism, except for the Juan Fernández islands because no information of phylogenetic value was available for this area. Four phylogenetic values of taxonomic weight were calculated, using standardization and endemicity. Results show that standardization of phylogenetic value produce data bias when cladograms differ in size. Complementarity between areas appears as a useful secondary tool. Nevertheless, the results show that complementarity must be used in conjunction with specific diversity and endemicity, to determine the importance of areas. Comparisons of results obtained using W, We, and strict and modified complementarity analyses showed that phylogenetic value with endemicity (We) can be applied as a unique value to determine the importance of each area. Using We, the six first areas selected, accumulated $72 \%$ of phylogenetic value, and $74 \%$ of gamma diversity of the southern South American carabids. None of the other used parameters accumulated $70 \%$ of species before the seventh area is added. The six selected areas are the Valdivian rain forest, pampean mountains, Coquimbo, western Patagonia, central Chile, and Araucanian. It is worth to noting that pampean mountains and western Patagonia are grassland habitats, Coquimbo and central Chile are arid and semiarid habitats, and only the Valdivian rain forests and Araucanian are forests with Nothofagus trees.
\end{abstract}

Key words: phylogenetic value, endemicity, complementarity, Carabidae, South America. 


\section{INTRODUCCIÓN}

La biodiversidad de América del Sur austral es de un alto valor, debido a su riqueza en especies que son filogenéticamente valiosas, como así también por estar amenazada ambientalmente (Morrone et al. 1996). Dinerstein et al. (1995) consideraron que desde el punto de vista de la conservación, América del Sur austral tiene una prioridad regional muy importante debido a su particularidad biológica. Esta está definida por varios aspectos, tales como la riqueza de especies, alto grado de endemicidad, particularidad de los procesos ecológicos y por su estado de conservación. Otro aspecto remarcable es la estrecha relación filogenética de su flora y fauna con las biotas de otras regiones australes, como Australia y Nueva Zelanda. Esta estrecha afinidad con biotas extraamericanas, y una menor afinidad con la biota neotropical llevan a que América del Sur austral sea considerada en numerosos esquemas biogeográficos como una región diferente al resto de América del Sur (Morrone 1996). Una de las áreas más fascinantes de esta región es el dominio subantártico, con tres principales ambientes, bosques fríos con especies dominantes de árboles del género Nothofagus Blume, regiones más cálidas con desiertos arbustivos en Chile central y pastizales fríos, tanto de baja altura como los de la Patagonia, como los de altura de la Puna.

Los criterios utilizados en América del Sur austral para crear áreas naturales protegidas ha sido en la mayoría de los casos la preservación de paisajes o de bosques. Debido al incremento de la actividad humana, es urgente la necesidad de estimar la proporción de especies protegidas como así también aquellas que deberían ser protegidas en el futuro. Generalmente el número total de especies es una de las medidas más usadas para hacer comparaciones entre áreas. Mediante la aplicación de este criterio cada especie constituye una unidad y todas las especies poseen el mismo valor, por lo que la pérdida de una especie tiene el mismo valor que la pérdida de cualquier otra. Otro criterio que se aplica es priorizar áreas en donde exista una alta concentración de especies endémicas. Debido a que la conservación de todas las áreas y todas las especies es imposible, son necesarias herramientas metodológicas para tomar decisiones con el fin de asegurar que se conserve la máxima diversidad posible (Morrone \& Crisci 1992). Durante la última década se han propuesto varios métodos para evaluar prioridades en conservación que incorporan la información filogenética (Humphries et al. 1991, Vane-Wrigth et al. 1991, Williams et al. 1991, Faith 1992, 2002). El método de Vane-Wrigth et al. (1991) es una herramienta práctica para asignar prioridades para la conservación de la biodiversidad en América del Sur austral, y ha sido aplicado por Morrone et al. (1996) para áreas templadas del Mundo. Vane-Wrigth et al. (1991) proponen un método que da prioridad a los taxa basales porque ellos son filogenéticamente raros, y crea un índice que toma en cuenta el número de clados en el cladograma en donde cada especie es incluida.

Se han realizado algunas contribuciones para América del Sur austral considerando el valor filogenético (Freire et al. 1998, Posadas et al. 2001). En estos trabajos el valor filogenético de cada especie es referido a áreas naturales en donde la especie está presente, las que se denominan áreas de endemismo (AE). El supuesto que las especies puedan ocupar toda el AE es usado sin cuestionar en casi la mayoría de los trabajos que utilicen dichas unidades areales. Si bien existe una gran posibilidad de que estas especies ocupen la mayoría del área de endemismo, el uso de estas unidades es realizado porque posibilita la comparación entre distintas especies que han sido colectadas en distintas localidades (Szumik \& Roig-Juñent en prensa). Esto es debido en gran medida a que el conocimiento de la riqueza de especies y distribución de los insectos es actualmente muy escaso, incluso para las áreas naturales protegidas (Roig-Juñent \& Claver 1999). Por ejemplo, para las 502 especies de carábidos (Insecta: Coleoptera) de América del Sur austral, 158 especies $(31 \%)$ han sido registradas en una localidad y 121 (24\%) en tan solo dos o tres localidades. Estas áreas endémicas han sido también evaluadas usando diferentes índices de importancia filogenética (Freire et al. 1998, Posadas et al. 2001), pero estos autores no han considerado las áreas montañosas extraandinas, las que poseen una alta diversidad y son ricas en especies endémicas (Roig-Juñent et al. 2003).

El valor filogenético, la diversidad gamma y el grado de endemismo, son todos paráme- 
tros útiles para caracterizar las áreas de endemismo, y deben ser considerados cuando se seleccionan áreas para la conservación. La complementariedad de especies entre áreas es muy útil porque permite establecer la máxima diferencia entre áreas y elegir aquellas que maximicen el número de especies protegidas. Sin embargo, un análisis estricto de complementariedad utilizando diversidad gamma no toma en cuenta otros parámetros como la importancia filogenética y el grado de endemismo.

En la presente contribución se propone una división geográfica de América del Sur austral en áreas de endemismos, incluyendo algunas de ellas que no habían sido tomadas en cuenta en trabajos previos (Morrone et al. 1994, Freire et al. 1998, Posadas et al. 2001, Roig-Juñent et al. 2003). Para estas áreas calculamos cuatro índices de valor filogenético utilizando la información cladística de cuatro de las tribus más representativas de Carabidae del área, así como la diversidad gamma y porcentaje de endemismo. La familia Carabidae es la cuarta en importancia por el número de especies para la región, con 502 especies. Además, la fauna de carábidos de América del Sur austral muestra un alto grado de endemismo, con 427 especies $(85 \%), 56$ géneros y siete tribus endémicas. Para establecer prioridades en relación con la conservación, discutimos la validez de algunos índices y comparamos diferentes métodos para seleccionar áreas a conservar. Se propone una nueva forma de usar la complementariedad con el objetivo de maximizar la cantidad de especies a conservar.

\section{MATERIALES Y MÉTODOS}

\section{América del Sur austral: definición y áreas na- turales}

Diferentes autores han definido América del Sur austral como el área al sur del paralelo $30^{\circ}$ $\mathrm{S}$, pero incluyendo regiones de altura de los Andes al norte de esta latitud (Jeannel 1967, Kuschel 1969, Crisci et al. 1991, Morrone et al. 1994). Sin embargo, América del Sur austral comprende un área continental continua en Chile bajo el paralelo $29^{\circ} \mathrm{S}$, el centro oeste de Argentina entre los parelelos 35-40응 S, y la Patagonia argentina al sur del paralelo $40^{\circ} \mathrm{S}$
(Fig. 1). Además, en Argentina existen sistemas montañosos extraandinos (áreas 13 a 16 en la Fig. 1), así como numerosas islas del Pacífico y Atlántico (Juan Fernández, San Ambrosio, Chiloé, Chonos y Cabo de Hornos en el Océano Pacífico, Isla de los Estados, Malvinas y Georgias del Sur en el Atlántico, como así también Tierra del Fuego con costas en ambos océanos) que también pertenecen a América del Sur austral.

Distintos taxa como así también distintas metodologías han sido utilizadas para proponer áreas de endemismo en América del Sur austral, aunque todas estas han sido siempre utilizando las áreas de distribución congruentes de varias especies (Morrone et al. 1994). Las 17 áreas de endemismo propuestas en esta contribución (Fig. 1) son aquellas propuestas en contribuciones previas que utilizaron distintos conjuntos de especies de artrópodos (Roig-Juñent 1994, 2002, Morrone et al. 1994, Roig-Juñent \& Flores 2001, Posadas et al. 2001). Estas áreas coinciden en general con aquellas propuestas como áreas biogeográficas (Cabrera \& Willink 1973).

\section{Diversidad gamma y especies endémicas}

El número de géneros y especies, así como la distribución de cada especie fue tomada de diferentes fuentes, tales como catálogos, descripciones originales, revisiones sistemáticas (ver Roig-Juñent 1998, Roig-Juñent \& Domínguez 2001) y de colecciones de museos e instituciones científicas. De un total de 502 especies, 21 de ellas se conocen solo para la localidad tipo, la cual es muy ambigua (solo se refiere como "Chile austral", "Patagonia" o por alguna provincia como Neuquén). Por esta razón, estas 21 especies no han sido tomadas en cuenta y se han utilizado un total de 481 especies.

\section{Comparación entre valores filogenéticos}

Para estimar el valor filogenético para cada área utilizamos la filogenia de diferentes taxa, donde los valores de información (I) reflejan el número de grupos a los cuales pertenece una especie (Fig. 4). El valor total $\mathrm{I}_{\mathrm{T}}$ (la suma de todos los valores de I de cada especie) es 


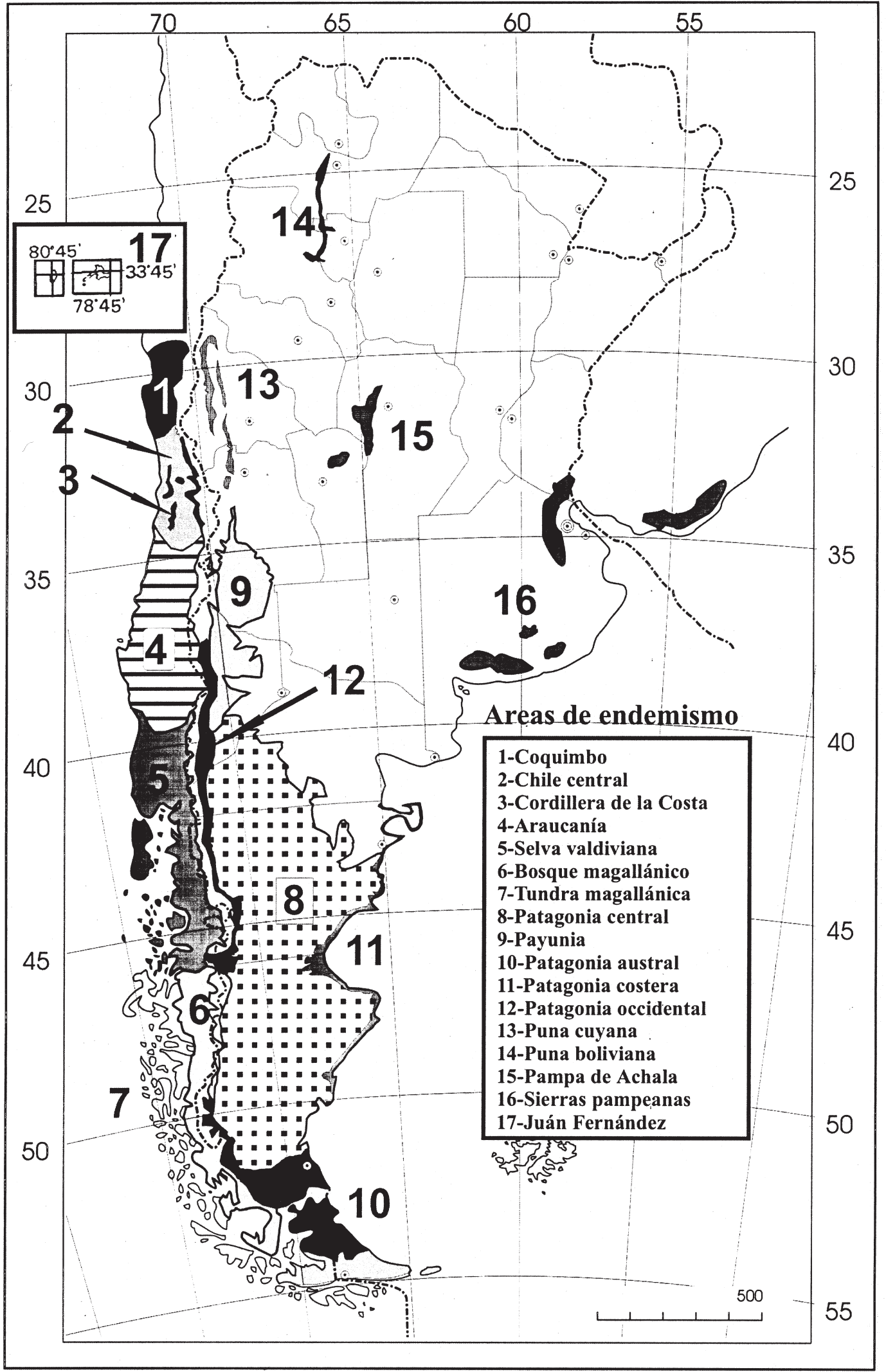

Fig. 1: Áreas de endemismo de América del Sur austral.

Areas of endemism of southern South America. 
dividido por el valor de I de cada especie, obteniendo el peso filogenético basal (Q) para esa especie. Luego, el valor Q se estandariza dividiéndolo por el menor valor de Q, obteniendo el valor filogenético (W). Esos valores de $\mathrm{W}$ constituyen un índice que muestra la proporción en la cual cada taxón contribuye a la diversidad total del grupo. El índice W puede también expresarse como porcentaje $(\mathrm{P})$. El valor calculado de $\mathrm{W}$ para cada especie que habita una región determinada puede ser asignado a esa área (Morrone et al. 1996). De esta manera, un área tendrá un valor filogenético (VF) igual a la suma de los valores de las especies que existen en ella, y las áreas pueden ser jerarquizadas por este VF. Cuando dos o más cladogramas son usados, el VF para cada área de endemismo es obtenido realizando una media entre los valores para esa área de cada uno de los cladogramas (Posadas et al. 2001, Roig-Juñent et al. 2001).

Posadas et al. (2001) han propuesto algunas modificaciones para el valor filogenético. Debido a que el índice W depende del cladograma en donde es calculado, estos autores proponen que es necesario estandarizar sus valores para evitar sesgos debido a la diferencia en la cantidad de taxa terminales. Esta estandarización, denominada Ws (Posadas et al. 2001), es realizada dividiendo el valor de $W$ de cada taxón en cada cladograma por la suma total de los valores de W.

Otra modificación del índice $\mathrm{W}$ que ha sido propuesta por Posadas et al. (2001) y RoigJuñent et al. (2001), consiste en darle más importancia a aquellas especies que tienen distribución restringida que a las que poseen una distribución más amplia. Estos autores consideran que el valor filogenético de una especie que ocupa más de un área de endemismo debe ser dividido por el número de áreas que esta especie ocupa y que ese valor resultante es el que debe ser sumado a cada una de las áreas ocupadas. Posadas et al. (2001) denominan a este nuevo índice como We, que implica que el valor del índice $\mathrm{W}$ es dividido por el número de áreas que el taxon ocupa, y Wes, que indica que el que es dividido es el valor del índice estandarizado Ws (Fig. 5).

\section{Filogenias utilizadas}

Se ha utilizado la información cladística de cuatro tribus (o subtribus) de carábidos endémicos de la región. Los taxa analizados son Cnemalobini con 28 especies (Roig-Juñent 2002), Broscina con 23 especies (Roig-Juñent 1995, 2000), Ceroglossini con siete especies, y Migadopini con 13 especies. Estas cuatro tribus, además de ser endémicas de la región, constituyen elementos distintivos de la fauna de América del Sur austral y están estrechamente relacionadas con otros taxa de Australia y Nueva Zelanda. Los análisis cladísticos de las tribus Cnemalobini y Broscini fueron tomados de Roig-Juñent (1995, 2000, 2002), mientras que aquellos de Migadopini y Ceroglossini fueron desarrollados para esta contribución y se muestran en las Fig. 2 y 3.

Comparación entre análisis de complementariedad estricta y modificada

Para obtener el índice de Jaccard (J) y el número de especies compartidas entre áreas de endemismo de América del Sur austral se utilizó el programa ESTIMATES 6.0b1 (Colwell 2000) y luego se calculó el complemento entre

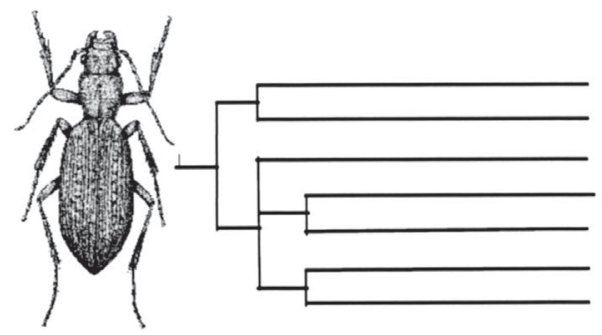

C. speciosus

C. suturalis

C. darwini

C. sybarita

C. valdiviae

C. intermedius

C. chilensis
Selva valdiviana; Bosque magallánico

Selva valdiviana; Bosque magallánico; Tundra magallánica Selva valdiviana

Selva valdiviana

Araucanía; Selva valdiviana; Bosque magallánico

Araucanía; Selva valdiviana

Araucanía; Selva valdiviana

Fig. 2: Cladograma de las especies de Ceroglossini, mostrando las áreas ocupadas por cada especie. 


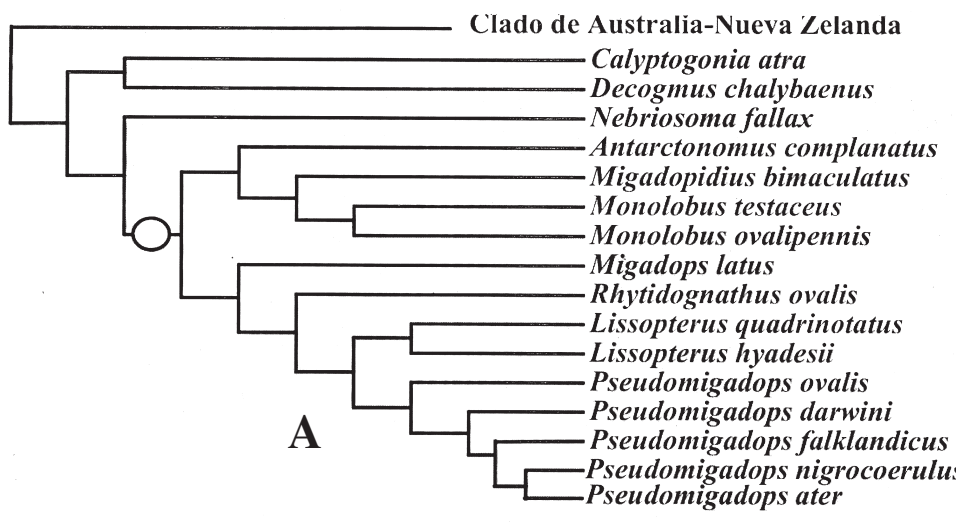

Australia- Nueva Zelanda

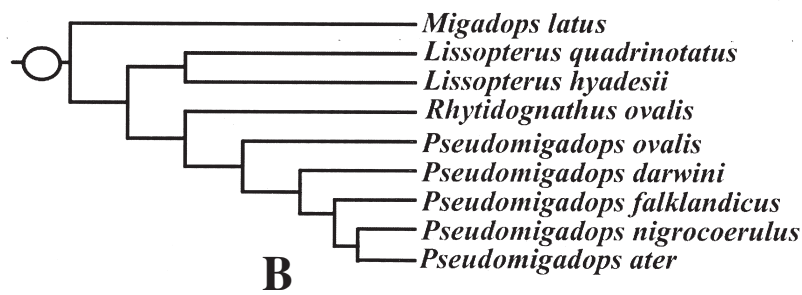

$\mathrm{AR}=$ Araucanía BM= Bosque magallánico PA= Patagonia austral $\mathrm{SP}=$ Sierras pampeanas $\mathrm{SV}=$ Selva valdiviana TM= Tundra magallánica

Fig. 3: Cladograma de las especies de Migadopini, mostrando las áreas ocupadas por cada especie. A y $\mathrm{B}$ representan las dos posibles resoluciones para el mismo clado.

Cladogram of Migadopini species, showing the areas occupied by each species. A and B are two different resolution of the same clade.

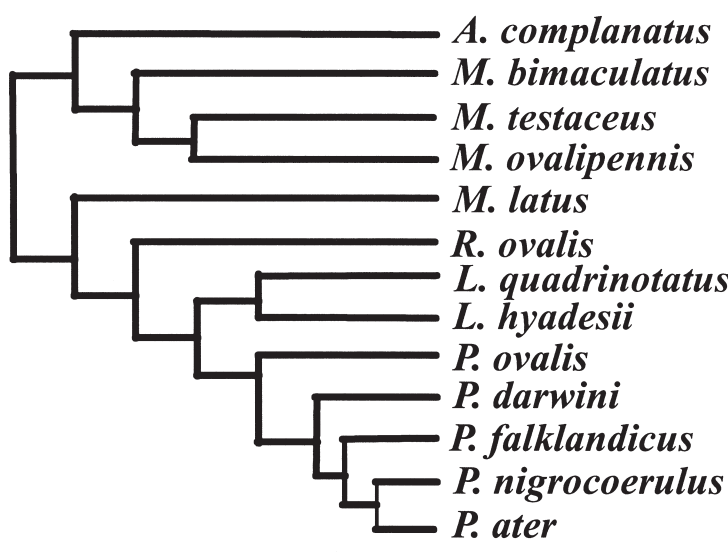

Cladograma de los Migadopini de América del Sur

\begin{tabular}{|l|r|r|r|c|}
\multicolumn{1}{c}{$\mathbf{I}$} & \multicolumn{1}{c}{$\mathbf{Q}$} & $\mathbf{W}$ & Porc. & Ws \\
\hline 2 & 31,00 & 4,00 & 15,03 & 0,150 \\
3 & 20,67 & 2,67 & 10,03 & 0,100 \\
4 & 15,50 & 2,00 & 7,51 & 0,075 \\
4 & 15,00 & 2,00 & 7,51 & 0,075 \\
2 & 31,00 & 4,00 & 15,03 & 0,150 \\
3 & 20,67 & 2,67 & 10,03 & 0,100 \\
5 & 12,40 & 1,60 & 6,01 & 0,060 \\
5 & 12,40 & 1,60 & 6,01 & 0,060 \\
5 & 12,40 & 1,60 & 6,01 & 0,060 \\
6 & 10,33 & 1,33 & 4,99 & 0,049 \\
7 & 8,86 & 1,14 & 4,28 & 0,042 \\
8 & 7,75 & 1,00 & 3,75 & 0,037 \\
8 & 7,75 & 1,00 & 3,75 & 0,037 \\
\hline 62 & & 26,61 & 100,1 & 1,000 \\
\hline
\end{tabular}

Fig. 4: Cálculo del peso taxonómico. La columna I indica el número de grupos a los cuales pertenece cada especie $(\mathrm{Ij}$ = número de grupos a los cuales pertenece el taxón j). La columna $\mathrm{Q}$ representa el peso filogenético basal $(\mathrm{Qj}=\mathrm{Si} / \mathrm{Ij})$. La columna W representa el valor filogenético y es la proporción a la cual el taxón j contribuye a la diversidad total del grupo $(\mathrm{Wj}=\mathrm{Qj} / \mathrm{Qmin})$. La columna Porc. es el porcentaje del valor W para cada taxón. La columna Ws representa el valor estandarizado del índice W de Posadas et al. (2001).

Calculation of phylogenetic value. Column I indicates the number of groups to which each species belongs $(\mathrm{Ij}=$ number of groups to which taxon $\mathrm{j}$ belongs). Column $\mathrm{Q}$ represent the basal phylogenetic weight $(\mathrm{Qj}=\mathrm{Si} / \mathrm{Ij})$. Column W represents the proportion to which taxon $\mathrm{j}$ contributes to the total diversity of the group $(\mathrm{Wj}=\mathrm{Qj} / \mathrm{Qmin})$. Column Porc. is the percentage of the $\mathrm{W}$ value for each taxon. Column Ws represents the value of the standardized W index of Posadas et al. (2001). 
áreas (1-J). Se utilizó la información de todas las especies presentes en la región excluyendo a 21 especies cuyos datos de distribución eran deficientes y a las especies endémicas del archipiélago Juan Fernández, ya que de esta última no se disponía de datos de valor filogenético. El total de especies usadas en este análisis fue de 462

Utilizando el mismo conjunto de datos se realizaron dos tipos de análisis. En primer lugar se realizó un análisis de complementariedad estricto tomando en consideración solo los valores más altos de complemento entre áreas. Varios autores (Vane-Wright et al. 1991, Freire et al. 1998, Posadas et al. 2001) proponen que luego de que la primer área es seleccionada por su VF, la segunda área que debe ser elegida para conservar debe ser aquella que muestra el mayor valor de complemento con respecto a la primera área. Sin embargo, cuando dos o más áreas muestran un valor de complemento similar, deberían tomarse en cuenta otros parámetros para seleccionar la siguiente área. En esta contribución, proponemos que cuando los valores de complemento sean similares se tome en cuenta también el valor filogenético, la diversidad gamma y la proporción de especies endémicas.

\section{RESULTADOS Y DISCUSIÓN}

\section{Diversidad gamma y grado de endemismo}

La Tabla 1 muestra las 17 áreas de endemismo ordenadas por la riqueza de especies que cada una posee, incluyendo además el tamaño del área y la cantidad de especies endémicas de ellas. Cuatro de estas áreas poseen un importante número de especies: Araucanía, selva valdiviana, sierras pampeanas y Chile central. Tres de ellas son contiguas y se ubican en Chile, entre los paralelos $32^{\circ}$ a $46^{\circ}$ de latitud sur. Se puede observar también un segundo grupo de áreas que poseen un importante número de especies, de 59 a 69, conformado por tres áreas: Coquimbo, Patagonia occidental y la Patagonia central. Las restantes áreas poseen menos de 50 especies. Es de hacer notar que algunas de las áreas más pobres en número de especies, muestran un alto valor de endemicidad. Por ejemplo, el archipiélago de Juan Fernández posee uno de los valores más bajos en número de especies, pero la mayoría de ellas (73\%) son endémicas. De la misma manera, áreas continentales como la Puna boliviana y la Patagonia austral tienen más del $40 \%$ de especies endémicas. Por el

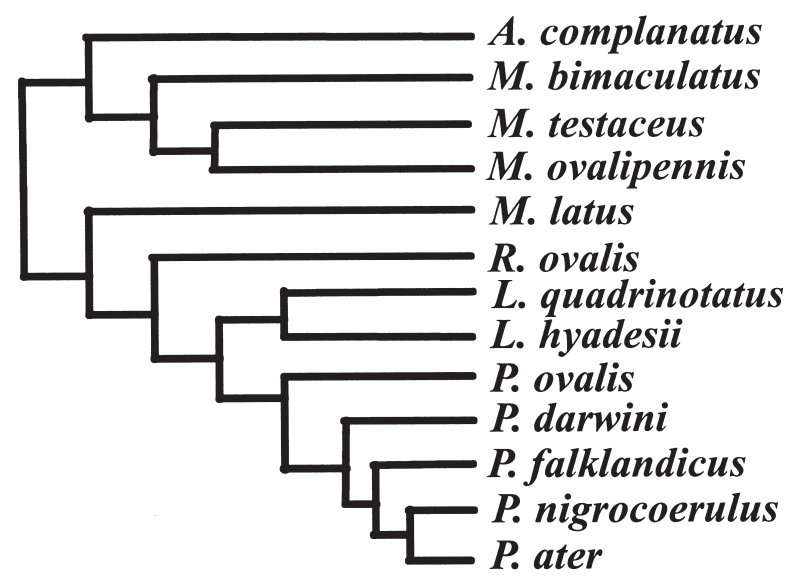

Áreas
\begin{tabular}{|l|l|l|l|l|}
\hline 3 & $\mathbf{W}$ & We & Ws & Wes \\
3 & 4,00 & 1,33 & 0,150 & 0,050 \\
1 & 2,67 & 0,89 & 0,100 & 0,033 \\
1 & 2,00 & 2,00 & 0,075 & 0,075 \\
3 & 4,00 & 2,00 & 0,075 & 0,075 \\
1 & 2,67 & 2,33 & 0,150 & 0,050 \\
2 & 1,60 & 0,80 & 0,100 & 0,100 \\
2 & 1,60 & 0,80 & 0,060 & 0,030 \\
2 & 1,60 & 0,80 & 0,060 & 0,030 \\
2 & 1,33 & 0,66 & 0,049 & 0,030 \\
1 & 1,14 & 1,14 & 0,042 & 0,042 \\
1 & 1,00 & 1,00 & 0,037 & 0,037 \\
1 & 1,00 & 1,00 & 0,037 & 0,037 \\
\hline
\end{tabular}

Fig. 5: Estimación de la endemicidad para el peso taxonómico. La columna W representa el índice W. La columna We representa el valor W dividido sobre el número de áreas ocupadas por la especie. La columna Ws representa la estandarización del índice y la columna Wes representa el valor de Ws dividido por el número de áreas que ocupa la especie.

Estimation of endemicity for the phylogenetic value. Column W represents the index W. Column We represents the value of $\mathrm{W}$ divided by the number of areas occupied by the species. Column Ws represents the standardized W index, and column Wes represents the value of Ws divided by the number of areas occupied by the species. 
contrario La Araucanía y la selva valdiviana con la mayor cantidad de especies de carábidos, tienen el $19 \%$ y $29 \%$ de especies endémicas respectivamente.

\section{Valor filogenético}

Los índices W, We, Ws y Wes fueron calculados para 16 áreas de endemismo de América del Sur austral, las que se encuentran ordenadas por su VF en la Tabla 2. El archipiélago de Juan Fernández no fue considerado por no poseer información filogenética.

Utilizando cualquiera de los índices W, el área con máximo valor es la selva valdiviana. Esta área posee la mayor cantidad de especies con VF conocido (13 especies, Tabla 1), un hecho que podría estar sesgando su valor. Sin embargo otras áreas con cantidades similares de especies con VF conocido, como la Araucanía y la Patagonia occidental (ambas con 11 espe- cies cada una), tienen un valor considerablemente más bajo, e incluso hay áreas con la mitad de especies con VF conocido, como las sierras pampeanas, que tiene un VF similar a la Araucanía y mayor que la Patagonia occidental (Tabla 2).

La siguiente área a seleccionar por su importancia dependerá de que índice $\mathrm{W}$ se utilice. En este punto, es necesario hacer algunas comparaciones de las ventajas y desventajas de cada índice. Posadas et al. (2001) proponen el uso de la estandarización del índice $\mathrm{W}$ para comparar árboles de diferentes tamaños, para evitar el alto peso que los árboles con muchas especies terminales tendrían sobre los que poseen menos. En realidad, esta estandarización del índice $\mathrm{W}$ estaría produciendo que los árboles con pocas especies posean un valor alto. Para ejemplificar este fenómeno compararemos dos cladogramas hipotéticos con diferente número de especies, el taxón A con 22 especies y el taxón B con tres especies (Fig. 6). Para el

TABLA 1

Diversidad gamma de las áreas de endemismo. El primer número representa el total conocido de especies, géneros y tribus para el área, después de la barra se representa el número de taxa endémicos. La última columna indica el número de especies para las que se conoce el valor filogenético (VF) y el porcentaje que representan en cada área

Gamma diversity for each endemism area. Figures before slash represents the total number of known species, genera, and tribes for each area. The number after the slash represents the endemic taxa. The last column indicates number of species with known phylogenetic value (VF) and the percentage that represent in each area

\begin{tabular}{|c|c|c|c|c|c|}
\hline Área de endemismo & Especies & Géneros & Tribus & $\begin{array}{l}\text { Superficie } \\
\left(10^{3} \mathrm{ha}\right)\end{array}$ & $\begin{array}{c}\text { Especies con } \\
\text { VF conocido }(\%)\end{array}$ \\
\hline Araucanía & $117 / 22$ & $51 / 3$ & 17 & 100.448 & $11 \quad(9,4)$ \\
\hline Selva valdiviana & $107 / 30$ & $49 / 4$ & 17 & 94.710 & $13(12,1)$ \\
\hline Sierras pampeanas & $104 / 34$ & $47 / 2$ & 17 & 30.710 & $6 \quad(5,7)$ \\
\hline Chile central & $97 / 26$ & $38 / 0$ & 15 & 26.765 & $6 \quad(6,1)$ \\
\hline Coquimbo & $69 / 13$ & $34 / 3$ & 12 & 25.900 & $7 \quad(10,1)$ \\
\hline Patagonia occidental & $60 / 11$ & $23 / 0$ & 9 & 24.800 & $11(18,3)$ \\
\hline Patagonia central & $59 / 5$ & $28 / 0$ & 12 & 418.380 & $5 \quad(8,4)$ \\
\hline Patagonia austral & $47 / 19$ & $23 / 1$ & 10 & 51.245 & $8(17,0)$ \\
\hline Cordillera de la Costa & $38 / 12$ & $20 / 1$ & 11 & 5.185 & $6(15,7)$ \\
\hline Bosque magallánico & $31 / 5$ & $24 / 0$ & 13 & 79.830 & $9(29,0)$ \\
\hline Patagonia costera & $30 / 5$ & $13 / 0$ & 7 & 15.060 & $5(16,6)$ \\
\hline Puna cuyana & $29 / 9$ & $18 / 1$ & 9 & 6.990 & $2 \quad(6,8)$ \\
\hline Puna boliviana & $28 / 13$ & $16 / 0$ & 8 & 4.600 & $2 \quad(7,1)$ \\
\hline Tundra magallánica & $27 / 9$ & $17 / 2$ & 9 & 50.880 & $7(25,9)$ \\
\hline Juan Fernández & $26 / 19$ & $12 / 0$ & 6 & 96 & - \\
\hline Payunia & $24 / 2$ & $15 / 0$ & 10 & 59.640 & $5(20,8)$ \\
\hline Pampa de Achala & $17 / 4$ & $11 / 0$ & 6 & 6.740 & $2(11,7)$ \\
\hline
\end{tabular}


TABLA 2

Valores de los diferentes índices de diversidad filogenética (W) calculados para cada una de las áreas de endemismo: Ws es el índice W estandarizado, We y Wes son el índice W y Ws corregidos por endemicidad, respectivamente (ver en el texto para el cálculo)

Values of different phylogenetic value indeces (W) calculated for each endemism area. Ws is the standardized W index, We and Wes represent the index W and Ws corrected by endemicity, respectively (see the text for details)

\begin{tabular}{lcccc}
\hline Área de endemismo & We & W & Ws área & Wes \\
\hline Selva valdiviana & 5,78 & 8,49 & 0,423 & 0,272 \\
Sierras pampeanas & 3,46 & 4,82 & 0,106 & 0,082 \\
Coquimbo & 3,05 & 3,92 & 0,070 & 0,055 \\
Patagonia occidental & 2,78 & 3,88 & 0,076 & 0,062 \\
Chile central & 2,27 & 3,13 & 0,061 & 0,046 \\
Araucanía & 2,10 & 5,06 & 0,174 & 0,084 \\
Bosque magallánico & 2,01 & 5,27 & 0,276 & 0,107 \\
Tundra magallánica & 1,76 & 4,08 & 0,184 & 0,032 \\
Pampa de Achala & 1,70 & 3,06 & 0,056 & 0,062 \\
Patagonia austral & 1,62 & 3,28 & 0,109 & 0,022 \\
Cordillera de la Costa & 1,55 & 2,09 & 0,040 & 0,024 \\
Puna boliviana & 1,25 & 1,25 & 0,024 & 0,023 \\
Payunia & 1,10 & 1,59 & 0,033 & 0,027 \\
Patagonia central & 1,06 & 1,82 & 0,039 & 0,024 \\
Patagonia costera & 1,06 & 1,81 & 0,037 & 0,009 \\
Puna cuyana & 0,41 & 0,55 & 0,011 & \\
\hline
\end{tabular}

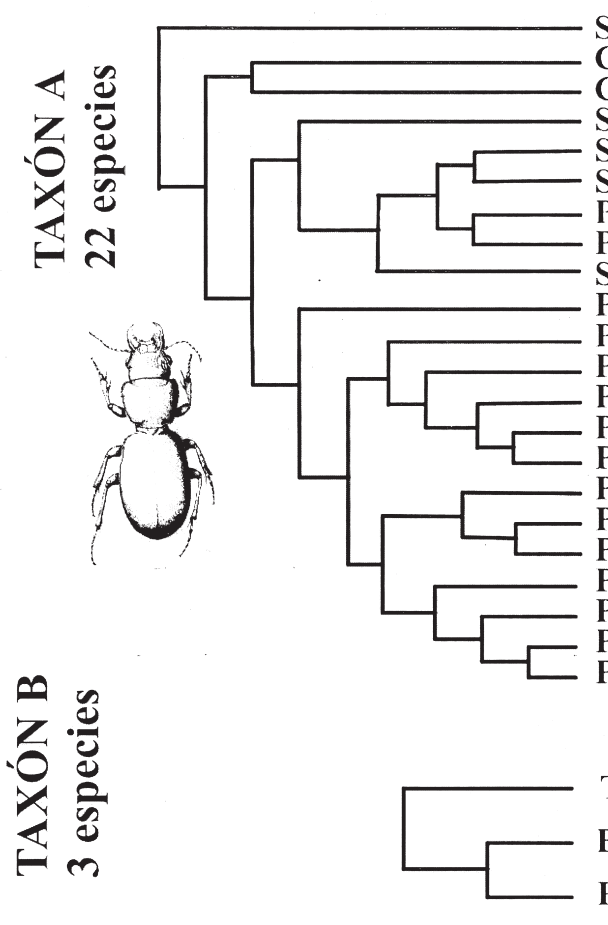

Selva valdiviana Chile central Cordillera de la Costa Sierras pampeanas Sierras pampeanas Sierras pampeanas Pampa de Achala Puna boliviana Sierras pampeanas Patagonia central Patagonia occidental

\begin{tabular}{|c|c|c|c|}
\hline I & $Q$ & $\mathbf{W}$ & Ws \\
\hline 1 & 152,00 & 10,00 & 0,244 \\
\hline 3 & 50,67 & 3,33 & 0,081 \\
\hline 3 & 50,67 & 3,33 & 0,081 \\
\hline 5 & 30,40 & 2,00 & 0,049 \\
\hline 8 & 19,00 & 1,25 & 0,031 \\
\hline 8 & 19,00 & 1,25 & 0,031 \\
\hline 8 & 19,00 & 1,25 & 0,031 \\
\hline 8 & 19,00 & 1,25 & 0,031 \\
\hline 6 & 25,33 & 1,67 & 0,041 \\
\hline 5 & 30,40 & 2,00 & 0,049 \\
\hline 7 & 21,71 & 1,43 & 0,035 \\
\hline 8 & 19,00 & 1,25 & 0,031 \\
\hline 9 & 16,89 & 1,11 & 0,027 \\
\hline 10 & 15,20 & 1,00 & 0,024 \\
\hline 10 & 15,20 & 1,00 & 0,024 \\
\hline 8 & 19,00 & 1,25 & 0,031 \\
\hline 9 & 16,89 & 1,11 & 0,027 \\
\hline 9 & 16,89 & 1,11 & $\mathbf{0 , 0 2 7}$ \\
\hline 8 & 19,00 & 1,25 & 0,031 \\
\hline 9 & 16,89 & 1,11 & 0,027 \\
\hline 10 & 15,20 & 1,00 & 0,024 \\
\hline 10 & 15,20 & 1,00 & 0,024 \\
\hline
\end{tabular}

Patagonia occidental

Patagonia occidental

Patagonia occidental

Patagonia occidental

Payunia

Patagonia austral

Patagonia austral

Patagonia central

Payunia

Patagonia costera
Patagonia central

15,20

0,024

Tundra magallánica

Bosque magallánico

Patagonia central

\begin{tabular}{|l|l|l|l|}
\hline 1 & 5,00 & 2,00 & 0,500 \\
2 & 2,50 & 1,00 & 0,250 \\
2 & 2,50 & 1,00 & 0,250 \\
\hline
\end{tabular}

Fig. 6: Cladogramas hipotéticos mostrando los problemas generados por el uso de la estandarización. Hypothetical cladograms showing the problems generated by the use of standardization. 
taxón A, la especie basal tiene un valor de Ws (el índice estandarizado $\mathrm{W}$ propuesto por Posadas et al. 2001) de 0,244, mientras que para el taxón B la especie basal tiene casi dos veces ese valor $(0,500)$. Una de las especies más apicales del taxón A tiene un valor de Ws de 0,024 , mientras que para el taxón B el valor es diez veces mayor $(0,250)$, similar al de la especie basal del taxón A. Cuando hacemos el promedio de los valores de Ws para las áreas de ambos cladogramas (Fig. 6), las tres áreas que ocupan las especies del taxón B (Tabla 3) muestran un VF muy superior a las del taxón A. Consecuentemente, el uso de la estandarización produce un efecto no deseable, ya que taxa con pocas especies poseen valores de Ws mayores que aquellos con muchas especies (Tabla 3). Además, cuando realizamos el promedio de Ws entre diferentes cladogramas, los resultados tienden a priorizar áreas con especies que están más cercanas a la base de un cladograma, que aquellas que muestran una gran distancia con el nodo basal. Entonces, si utilizamos el índice Ws para los datos de Carabidae, la estandarización le dará excesivo peso al cladograma de Ceroglossini (que posee siete especies) con respecto a los cladogramas de Broscini y Cnemalobini (con 22 y 28 especies, respectivamente). Como las especies de Ceroglossini están distribuidas principalmente en la Selva Valdiviana, este artefacto de la estandarización podría ex- plicar el alto valor de Ws que posee esta área con respecto a la segunda en importancia, cuatro veces superior, mientras que aplicando $\mathrm{W}$ no llega al doble (Tabla 2).

Otra modificación del índice $\mathrm{W}$ es la endemicidad. La base de esta propuesta es que las especies restringidas en distribución (a veces también poco frecuentes) deben ser más importantes en el momento de priorizar áreas que aquellas que posean una distribución más amplia. Si comparamos cladogramas de igual cantidad de especies, como el de Barypina y Cnemalobini, podemos notar que la especie basal de Barypina, Microbarypus sylvatica Roig-Juñent, es una especie restringida en distribución, mientras que la basal de Cnemalobini, Cnemalobus striatus Waterhouse, es una especie muy común y ampliamente distribuida (Roig-Juñent 2000, 2002). Cuando utilizamos el índice W, la segunda y tercer área en importancia son el Bosque magallánico y la Araucanía (Tabla 2), mientras que si tomamos en cuenta la endemicidad (We) estas áreas son menos importantes y otras que poseen un gran número de especies endémicas adquieren mayor importancia (como las sierras pampeanas, Coquimbo y la Patagonia occidental; (Tabla 2).

Debido a que la estandarización produce un gran sesgo para seleccionar áreas por su importancia filogenética, decidimos utilizar el índice $\mathrm{W}$, y porque consideramos importante incluir la

TABLA 3

Valores filogenéticos estandarizados (Ws) para las áreas de acuerdo a dos taxa hipotéticos (ver Fig. 6)

Standardized phylogenetic values (Ws) for the considered areas according to two hypothetic taxa (see Fig. 6)

\begin{tabular}{|c|c|c|c|}
\hline Área & Taxón A & Taxón B & Media \\
\hline Tundra magallánica & - - & 0,500 & 0,250 \\
\hline Patagonia central & 0,104 & 0,250 & 0,177 \\
\hline Bosque magallánico & - & 0,250 & 0,125 \\
\hline Selva valdiviana & 0,244 & - & 0,122 \\
\hline Sierras pampeanas & 0,152 & - & 0,076 \\
\hline Patagonia occidental & 0,141 & - & 0,070 \\
\hline Cordillera de la Costa & 0,081 & - & 0,040 \\
\hline Chile central & 0,081 & 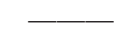 & 0,040 \\
\hline Patagonia austral & 0,054 & - - & 0,027 \\
\hline Payunia & 0,058 & - & 0,023 \\
\hline Pampa de Achala & 0,031 & - & 0,015 \\
\hline Puna boliviana & 0,031 & - - & 0,015 \\
\hline Patagonia costera & 0,024 & - & 0,012 \\
\hline
\end{tabular}


endemicidad en un área, decidimos aplicar este parámetro al índice $\mathrm{W}$ y ordenar las 16 áreas de acuerdo al índice We (Tabla 2).

\section{Análisis de complementariedad estricto y modi-} ficado

Realizando un análisis estricto de complementariedad entre áreas, el orden de importancia de ellas sería el que se observa en la Tabla 4. Por este método, debemos considerar como segunda área en importancia a la Patagonia costera, que posee el valor más alto de complemento $(0,99)$ con respecto a la selva valdiviana. Sin embargo, hay numerosas áreas con valor similar de complemento, como la puna boliviana y pampa de Achala $(0,98)$, y cinco áreas más con un alto valor de complemento $(0,96)$ (Tabla 4). Mientras que la Patagonia costera tiene 30 especies, cinco de ellas endémicas y comparte dos especies con la selva valdiviana, existen áreas como las sierras pampeanas, que poseen menor valor de complemento que la Patagonia costera $(0,96)$ con respecto a la selva valdiviana, pero que es tres veces más rica en especies, posee cerca de siete veces más especies endémicas y tiene tres veces más de VF. De acuerdo a esto, el uso del complemento estricto pone en segundo término áreas con alta importancia a favor de áreas con baja diversidad, endemicidad y valor filogenético. Esta diferencia del $3 \%$ en el complemento no justifica la elección de la Patagonia costera como segunda área de importancia. Por ello, proponemos considerar un conjunto adicional de parámetros cuando los valores de complementariedad sean semejantes. Estos parámetros son en orden de importancia: VF, diversidad gamma y cantidad de especies endémicas. Después de cada paso del análisis, el área con mayor valor de complemento es seleccionada junto con un conjunto de áreas que difieran en menos del $5 \%$ de ese valor. El área de mayor valor es elegida como siguiente en importancia, siempre y cuando no exista dentro del grupo de áreas de menor valor una que la supere en dos o más veces en $\mathrm{VF}$, o que duplique su diversidad gamma y el número de especies endémicas. Si estos tres parámetros se cumplen, el área que posee estas características será seleccionada como la siguiente en importancia.

Siguiendo esta metodología, la segunda área en importancia son las sierras pampeanas (Tabla 4), que muestra una alta disimilitud con

TABLA 4

Jerarquización de las áreas de acuerdo al valor filogenético We exclusivamente, aplicando completentaridad estricta y complementaridad modificada

Hierarchy of endemism areas according to We index, to strict complementarity, and modified complementarity

\begin{tabular}{llll}
\hline & We & Complementaridad estricta & Complementaridad modificada \\
\hline 1 & Selva valdiviana & Selva valdiviana & Selva valdiviana \\
2 & Sierras pampeanas & Patagonia costera & Sierras pampeanas \\
3 & Coquimbo & Puna boliviana & Patagonia occidental \\
4 & Patagonia occidental & Tundra magallánica & Tundra magallánica \\
5 & Chile central & Pampa de Achala & Puna boliviana \\
6 & Araucanía & Puna cuyana & Patagonia costera \\
7 & Bosque magellánico & Cordillera de la Costa & Pampa de Achala \\
8 & Tundra magallánica & Payunia & Coquimbo \\
9 & Pampa de Achala & Patagonia austral & Cordillera de la Costa \\
10 & Patagonia austral & Sierras pampeanas & Payunia \\
11 & Cordillera de la Costa & Coquimbo & Puna cuyana \\
12 & Puna boliviana & Patagonia occidental & Patagonia austral \\
13 & Payunia & Bosque magallánico & Bosque magallánico \\
14 & Patagonia central & Patagonia central & Patagonia central \\
15 & Patagonia costera & Chile central & Chile central \\
16 & Puna cuyana & Araucanía & Araucanía
\end{tabular}


la selva valdiviana, comparte con ella ocho especies, posee un alto valor de complemento y VF, y además posee una alta diversidad gamma y gran cantidad de especies endémicas (Tabla 1).

Ahora, necesitamos conocer la complementariedad entre las dos áreas elegidas y las restantes áreas. Para ello, se realiza un nuevo análisis considerando todas las especies presentes en las dos áreas seleccionadas (selva valdiviana y sierras pampeanas), comparándolos con las especies presentes en las 14 áreas restantes. Siguiendo el mismo método descrito anteriormente, podemos elegir una nueva área que maximice la diversidad y el VF. Esta jerarquía en importancia de las áreas, es la que se muestra en la Tabla 4.

\section{Comparación de la acumulación de especies y VF aplicando los distintos métodos}

Cuando ordenamos las áreas de endemismo de acuerdo al valor de We, las dos primeras áreas acumulan el $44 \%$ del total de especies consideradas, y el $40 \%$ del total de la información filogenética (índice W). Se llega al $74 \%$ de la diversidad gamma cuando se adiciona la sexta área (Fig. 7A), y estas seis áreas de América del Sur austral son las más ricas en cantidad de especies (Tabla 1). Observamos que estas seis primeras áreas son también las que podrían elegirse por su diversidad gamma. Sin embargo, el índice We nos proporciona un orden de importancia distinto al obtenido solo con diversidad gamma (Tabla 1). Altos valores de diversidad no siempre se corresponden con altos valores de We, y no hay concordancia en los resultados después de que la sexta área es elegida, indicando que la diversidad por sí misma no es un buen indicador del VF.

Utilizando el análisis de complementariedad modificado, las dos primeras áreas elegidas son las mismas que utilizando solo el valor de We (Fig. 7C). Sin embargo, luego que la sexta área es adicionada, solo el $64 \%$ del número de especies y el $67,7 \%$ de VF es alcanzado. El $75 \%$ de diversidad gamma es obtenido recién cuando se suman ocho a nueve áreas (Fig. 7C).

La acumulación de especies es menor cuando se utiliza un análisis de complementariedad estricto, ya que el $75 \%$ de la diversidad gamma es alcanzado cuando se han sumado 10-11 áreas (Fig. 7D). Siguiendo este método, después de la adición de la sexta área obtenemos solo el 44,8 \% de representación de las especies y el $54,8 \%$ del VF.

\section{CONCLUSIONES}

El uso de la estandarización no es recomendable debido a que tiende a asignar alto peso filogenético a taxa que poseen pocas especies. El uso de la endemicidad, por el contrario es recomendable ya que nos permite asignar mayor valor filogenético a áreas que poseen taxa exclusivos de ellas.

En el proceso de elección de áreas por su importancia, el uso de la complementariedad en forma estricta no maximiza el valor filogenético acumulado, mejores resultados son obtenidos realizando una jerarquía de áreas aplicando el índice We o la complementariedad con ciertas restricciones.

Empleando los carábidos como herramienta para determinar prioridades de conservación en la región austral de América del Sur, podemos concluir de acuerdo al índice We que la selva valdiviana es el área con mayor prioridad de conservación. Este resultado es concordante con el obtenido por Freire et al. (1998) quienes emplearon el índice $\mathrm{W}$ para varios taxa vegetales y animales. Esta área posee un número importante de taxa de Carabidae de alto rango que son endémicos, tales como la tribu Systolosomini y numerosos géneros relacionados con la fauna de Australia y Nueva Zelanda. El uso del índice We permitió identificar como área en segunda importancia a las sierras pampeanas. Esta área posee también numerosos géneros relictuales e incluso una tribu endémica de Carabidae, Notiokasini (Kavanaugh \& Nègre 1983), mostrando su biota una peculiar afinidad con la existente en África del Sur (De la Sota 1967) y otras regiones pangéicas de América del Sur (Mattoni \& Acosta 1997). La tercer área en importancia es un desierto con numerosos géneros endémicos de insectos que son relictuales y constituye la región más septentrional donde la flora subantártica permanece como relictos (e.g., Parque Nacional Fray Jorge). La cuarta área es la estepa patagónica más húmeda, la $\mathrm{Pa}$ tagonia occidental, donde el límite occidental está marcado por los bosques de Nothofagus. Chile central es la quinta área en importancia, a 


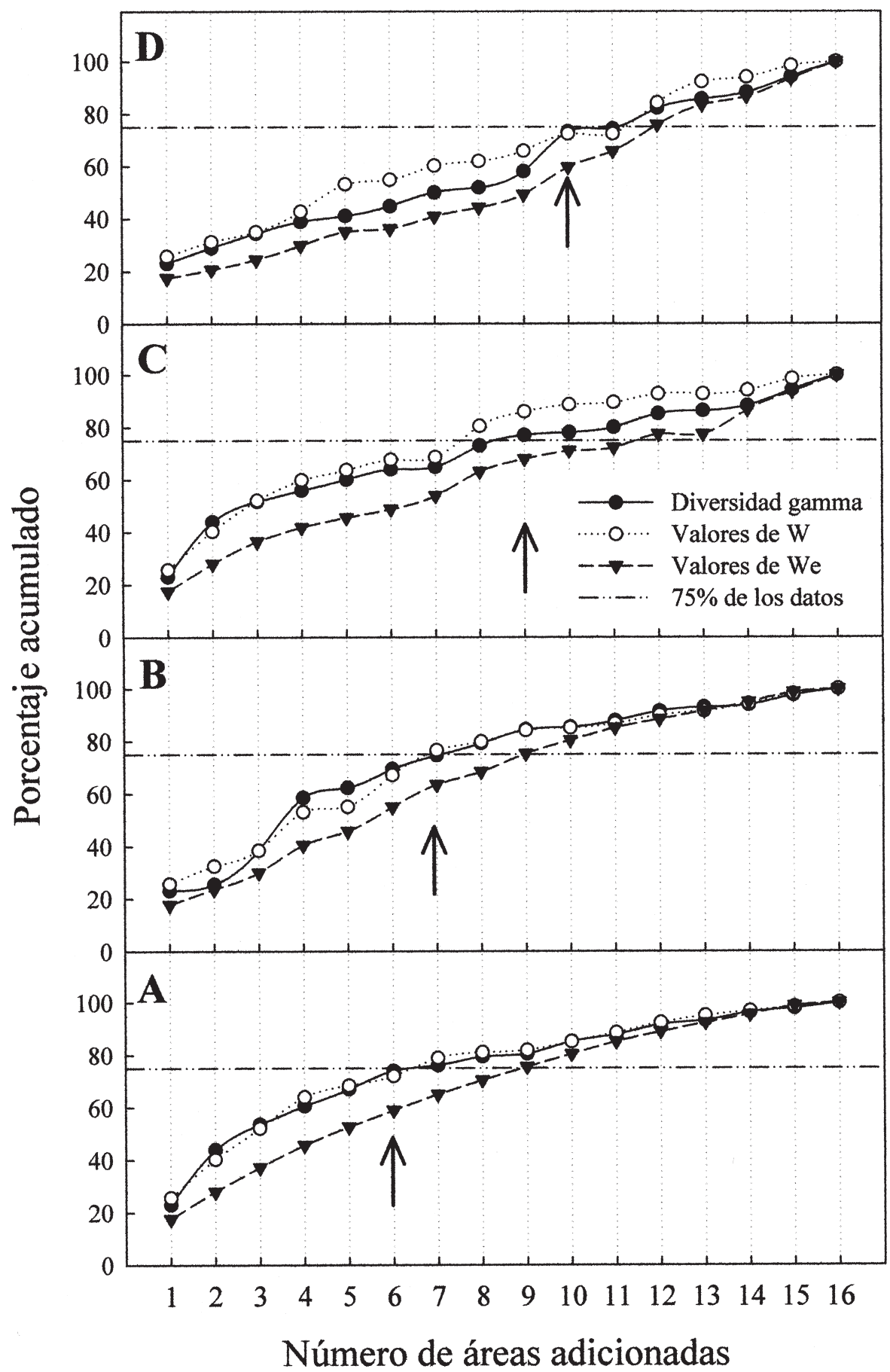

Fig. 7: Porcentaje de acumulación de diversidad gamma y del valor filogenético (W y We) cuando se adicionan áreas de endemismo. Los diferentes criterios para seleccionar áreas son la jerarquía obtenida utilizando el índice: (A) We, (B) W, (C) con complementariedad modificada, y (D) con complementariedad estricta. Para los números de áreas adicionadas ver tabla 4. Las flechas indican cuándo se alcanza el $75 \%$ de la diversidad gamma.

Accumulates percentage of gamma diversity and the phylogenetic value (W and We) when adding endemic areas. Different criteria used to select priority areas are: (A) the ranking obtained with We, (B) with $\mathrm{W}$, (C) with modified complementarity, and (D) with strict complementarity. For the numbers of endemic areas added see Table 4 . Arrows indicate $75 \%$ of gamma diversity. 
pesar de que Posadas et al. (2001) proponen esta área como la que tiene el mayor VF de acuerdo a su análisis. Esta diferencia de resultados es probablemente debido al uso de la estandarización en el índice W.

\section{AGRADECIMIENTOS}

Deseamos agradecer al Consejo Nacional de Investigaciones Científicas y Técnicas (CONICET, Argentina) por su apoyo y a las sugerencias de los dos árbitros anónimos realizaron sobre el trabajo. Este trabajo forma parte del proyecto "Diseño de una red de reservas para la protección de la biodiversidad en América del Sur Austral utilizando modelos predictivos de distribución con taxones hiperdiversos" de la Fundación BBVA.

\section{LITERATURA CITADA}

CABRERA A \& A WILLINK (1973) Biogeografía de América Latina. Monografía 13. Serie Biología, Organización de Estados Americanos. 117 pp.

COLWELL RK (2000) ESTIMATES 6.0b1, Statistical estimation of species richness and shared species from samples. http//viceroy.eeb.uconn.edu/ estimates.

CRISCI JV, MM CIGLIANO, JJ MORRONE \& S ROIGJUÑENT (1991) Historical biogeography of southern South America: a cladistic approach. Systematic Zoology 40: 152-171.

DE LA SOTA ER (1967) Composición, origen y vinculaciones de la flora pteridológica de las sierras de Buenos Aires (Argentina). Boletín de la Sociedad Argentina de Botánica 11: 105-128.

DINERSTEIN E, DM OLSON, DJ GRAHAM, AL WEBSTER, SA PRIMM, MP BOOKBINDER \& G LEDEC (1995) Una evaluación del estado de conservación de las ecoregiones terrestres de América Latina y el Caribe. World Wildlife Foundation and World Bank, Washington, District of Columbia, USA.

FAITH DP (1992) Conservation evaluation and phylogenetic diversity. Biological Conservation 61: $1-10$.

FAITH DP (2002) Quantifying biodiversity: a phylogenetic perspective. Conservation Biology 16: 249-252.

FREIRE SE, JJ MORRONE \& JV CRISCI (1998) Cladistic measures for conservation in Southern South America: a view from the Asteraceae (Angiosperms) and Curculionidae (Insecta: Coleoptera). Physis (Sección C) (Argentina) 55: 27-32.

JEANNEL R (1967) Biogeográphie de l'Amérique Australe. En: Delamare-Deboutteville C \& E Rappoport (eds) Biologie de l'Amérique Austral 2: 401-460. CNRS et CNICT, Paris, France.

KAVANAUGH DH \& J NĖGRE (1983) Notiokasiini- A new tribe of Carabidae (Coleoptera) from southeastern South America. Coleopterists Bulletin 36: $549-566$
KUSCHEL G (1969) Biogeography and ecology of South American Coleoptera. En: Fittkau EJ, J Illies, H Klinge, GH Schwabe \& H Sioli (eds) Biogeography and ecology in South America: 709-722. Junk Publishers, The Hague, The Netherlands.

HUMPHRIES CJ, RI VANE-WRIGHT \& PH WILLIAMS (1991) Biodiversity reserves: setting new priorities for the conservation of wildlife. Parks 2: 34-38.

MATTONI CI \& LE ACOSTA (1997) Scorpions of the insular sierras in the Llanos District (Province of La Rioja, Argentina) and their zoogeographical links. Biogeographica 73: 67-80.

MORRONE JJ (1996) Austral biogeography and relict weevil taxa (Coleoptera: Nemonychidae, Belidae, Brentidae, and Caridae). Journal of Comparative Biology 1: 123-127.

MORRONE JJ \& JV CRISCI (1992) Aplicación de métodos filogenéticos y panbiogeográficos en la conservación de la diversidad biológica. Evolución Biológica 6: 53-66.

MORRONE JJ, L KATINAS \& JV CRISCI (1996) On temperate areas, basal clades and biodiversity conservation. Oryx 30: 187-194.

MORRONE JJ, S ROIG-JUÑNENT \& JV CRISCI (1994) Cladistic biogeography of terrestrial subantarctic beetles (Insecta: Coleoptera) from South America. National Geographic Research and Exploration 10: 104-115.

MORRONE JJ, S ROIG-JUNENT \& GE FLORES (2002) Delimitation of biogeographic districts in central Patagonia (sothern South America), based on beetle distributional patterns (Insecta: Coleoptera: Carabidae and Tenebrionidae). Revista del Museo Argentino de Ciencias Naturales (Argentina) 4: 1-6.

POSADAS P, DR MIRANDA ESQUIVEL \& JV CRISCI (2001) Using phylogenetic diversity measures to set priorities in conservation: an example from Southern South America. Conservation Biology 15: 1325-1334.

ROIG-JUÑENT S (1994). Historia Biogeográfica de América del Sur Austral. Multequina (Argentina) 3: 167-203.

ROIG-JUÑENT S (1995) Cladistic analysis of Barypus Dejean 1828 (Coleoptera: Carabidae: Broscini). American Museum Novitates 3117: 1-11.

ROIG-JUÑENT S (1998) Carabidae. En: Morrone JJ \& S Coscarón (eds) Biodiversidad de artrópodos argentinos, un enfoque biotaxonómico: 194-209. Ediciones Sur, La Plata, Argentina.

ROIG-JUÑENT S (2000) The subtribes and genera of the tribe Broscini (Coleoptera: Carabidae): cladistic analysis, taxonomic treatment, and biogeographical considerations. Bulletin of the American Museum 255: 1-98.

ROIG-JUÑENT S (2002) Nuevas especies de Cnemalobus Guérin-Ménéville (Coleoptera, Carabidae) y consideraciones filogenéticas y biogeográficas sobre el género. Revista de la Sociedad Entomológica Argentina 61: 51-72.

ROIG-JUÑENT S \& S CLAVER (1999) La entomofauna del Monte y su conservación en las áreas naturales protegidas. Revista de la Sociedad Entomológica Argentina 58: 117-127.

ROIG-JUÑENT S, G FLORES, S CLAVER, G DEBANDI \& A MARVALDI (2001) Monte Desert (Argentina): insect biodiversity and natural areas. Journal of Arid Environments 47: 77-94.

ROIG-JUÑENT S \& C DOMÍNGUEZ (2001) Diversidad de la familia Carabidae (Coleoptera) en Chile. Revista Chilena de Historia Natural 74: 549-571. 
ROIG JUÑENT S \& GE FLORES (2001) Historia Biogeográfica de las áreas áridas de América del Sur austral. En: Morrone JJ \& J Llorente-Bousquets (eds) Introducción a la biogeografía en Latinoamérica: teorías, conceptos, métodos y aplicaciones: 257-266. Las Prensas de Ciencias, Facultad de Ciencias, Universidad Nacional Autónoma de México, México, Distrito Federal.

ROIG JUÑENT S, GE FLORES \& C MATTONI (2003) Consideraciones biogeográficas de la Precordillera (Argentina) basadas en artrópodos epígeos. En: Morrone JJ \& J Llorente-Bousquets (eds) Una perspectiva latinoamericana de la Biogeografía: 275288. Las Prensas de Ciencias, Facultad de Ciencias, Universidad Nacional Autónoma de México, México, Distrito Federal.

Editor Asociado: Pablo Marquet

Recibido el 16 de enero de 2004; aceptado el 19 de julio de 2004
VANE-WRIGHT RI, CJ HUMPHRIES \& PH WILLIAMS (1991). What to protect? Systematics and the agony of choice. Biological Conservation 55: 235-254.

WILLIAMS PH, CJ HUMPHRIES \& RI VANE-WRIGHT (1991) Measuring biodiversity: taxonomic relatedness for conservation priorities. Australian Systematic Botany 4: 665-667.

SZUMIK C \& S ROIG-JUÑENT (en prensa) Criterio de optimalidad para áreas de endemismo. Aplicación para América del Sur Austral. En: Morrone JJ \& J Llorente-Bousquets (eds) Una perspectiva latinoamericana de la Biogeografía. Las Prensas de Ciencias, Facultad de Ciencias, Universidad Nacional Autónoma de México, México, Distrito Federal. 